\title{
De las redes de colaboración para la innovación al desarrollo de clusters de ciencia y tecnología. Dos casos de estudio en la Universidad Nacional de San Martín en Argentina
}

Pablo Lavarello*

Mariana Minervini**

Verónica Robert***

Recebido: 22/11/2016 Versão Revisada (Entregue): 14/03/2017 Aprovado: 25/03/2017

\section{RESUMEN}

En este artículo discutimos las políticas orientadas a la promoción de clusters de ciencia y tecnología. En un cluster en formación vinculado a una universidad pública localizada en el conurbano de Buenos Aires, analizamos dos redes de cooperación desarrolladas bajo esquemas diferentes de políticas: bottom-up (kits diagnósticos para salud humana y animal) y top-down (diseño y caracterización de microdispositivos electrónicos). Adoptamos el enfoque de proximidades que permite caracterizar las tensiones entre oportunismo y encerramiento presentes en estas redes de cooperación. La metodología propuesta nos permite comparar cómo la red enfrenta estas tensiones bajo esquemas diferentes. Los resultados muestran que: ante una proximidad organizacional baja, con alto riesgo de oportunismo (bottom-up), la tendencia es a compensar con mayor proximidad social y mayor estabilidad a partir de usuarios líderes;

* Centro de Estudios Urbanos Regionales (Consejo Nacional de Investigaciones Científicas y Técnicas) y Universidad Nacional de San Martín (UNSAM), Buenos Aires, Argentina. Email: plavarel@gmail.com.

** Comisión de Investigaciones Científicas, Universidad Nacional de San Martín (CIC/UNSAM), Buenos Aires, Argentina. E-mail: marianaminervini03@gmail.com.

*** Consejo Nacional de Investigaciones Científicas y Técnicas, Universidad Nacional de San Martín (CONICET/UNSAM), Buenos Aires, Argentina. E-mail: vrobert@unsam.edu.ar. 
y ante una proximidad organizacional y tecnológica alta, con riesgo de encerramiento (topdown), la tendencia es de mayor apertura a nuevos usuarios.

Palabras Claves | Proximidades; redes de Cooperación; Políticas Bottom-up y Top-down

Códigos JEL | B52; O30; O38; R10

\section{From collaboration networks for innovation to the development of science and technology clusters. Two case studies at the National University of San Martín in Argentina}

\section{ABSTRACT}

In this article, we propose to discuss public policies oriented to promote science and technology clusters. For this purpose, we studied a particular cluster case linked to a public university located in the suburban area of the city of Buenos Aires: the National University of San Martín (UNSAM). We analyze two cooperation networks between UNSAM research groups, companies, technology centers and potential adopters of innovations: a "bottom up" cluster experience of diagnostic kits development and a "top-down" University-State experience, aero spatial micro-wave development. We adopt a "proximity approach" (RALLET; TORRE, 1999, 2005; BOSCHMA, 2005; KNOBEN, 2008; LAGENDIJK; OINAS, 2005). This approach goes beyond geographical proximity explanation of clusters coordination. It takes in account social proximity (GRANOVETTER, 1985), organizational proximity (Williamson, 1975, 1979, 1985), institutional proximity (EDQUIST; JOHNSON, 1997; HOFSTEDE, 1991) and technological proximity (COHEN; LEVINTHAL, 1990; NOOTEBOOM et al., 2007). This approach enables us to analyze the tensions between opportunism and lock-in that characterize university - enterprise relations and that should be taken into account when defining science and technology clusters. The results of the case studies show that these tensions are solved in an idiosyncratic and particular way. When organizational proximity is low and risks of opportunism are high (under a bottom-up scheme), the tendency is to compensate with greater social proximity. When organizational and technological proximity is high and there is a risk of lock in (under a top-down scheme), the tendency is to seek greater openness of the network with new applications and potential new users.

KeYwords | Proximity; Networks; Bottom Up and Top Down Policies

JEL CODES | B52; O30; O38; R10 


\section{Introducción}

Los clusters definidos en un sentido amplio comprenden un conjunto de relaciones de mercado no mercantiles entre organizaciones co-localizadas. Según la definición de Maskell (2001), permiten la creación de conocimiento a través de la variación y la profundización de la división del trabajo. También se ha señalado (ANTONELLI, 1992, 2000) la importancia de las diferentes externalidades derivadas del aprendizaje y la innovación. Otros trabajos (LUNDVALL 1992, 2007; FREEMAN, 1991) ponen de relieve la relevancia de las interacciones en los procesos de aprendizaje, debido a los componentes tácitos del conocimiento que se ponen en juego en las relaciones proveedor-cliente. La temática de los clusters de ciencia y tecnología (CCT) se encuentra estrechamente ligada a las dinámicas de aprendizaje colectivo y la innovación (KEEBLE; WILKINSON, 1999) y han hecho foco en casos que involucran fuertes relaciones universidad-empresa.

Existe una extensa literatura que ha analizado, desde diferentes perspectivas, experiencias de formación de clusters de ciencia y tecnología en países desarrollados (SAXENIAN, 1990, 1996; ARTHUR, 1990; BRESNAHAN; GAMBARDELLA; SAXENIAN, 2005; SCHMITZ, 1992; COOKE; MORGAN, 1994; LONGHI, 1999; NIOSI; BANIK, 2005; COLYVAS, 2007). Algunos trabajos han ampliado esta discusión a los casos en los países en desarrollo (BELL; ABU, 1999; LASTRES et al., 2003; CASSIOLATO, et al., 2002, REID-HENRY, 2006; BIANCHI, et al. 2011; VAIDYANATHAN, 2008; YOGUEL; BOSCHERINI, 1998, 2001), aunque, debido a la debilidad de los sistemas de ciencia y tecnología en estos países y su imbricación en sus estructuras productivas, los casos para países en desarrollo incluyen sistemas territoriales definidos de una forma amplia, considerando desde aglomeraciones industriales a sistemas territoriales complejos, con pocos aportes específicos a CCT.

Los clusters de ciencia y tecnología vinculados a universidades son un fenómeno novedoso en países en desarrollo y por lo tanto su estudio es relevante tanto para conocer su especificidad como para el diseño de políticas que aliente su desarrollo. En este trabajo, nos proponemos estudiar un caso particular de cluster emergente que tiene como origen la Universidad Nacional de San Martín (UNSAM), con una base de conocimientos en tecnologías genéricas como la biotecnología y la nanotecnología. Dada la localización de la Universidad en el centro de un tejido industrial altamente denso, la transferencia tecnológica, la incubación de empresas basadas en conocimiento y la promoción spin-offs universitarios, constituyen una 
parte central del quehacer universitario, junto a la investigación y la docencia. Este foco en las actividades de transferencia y la cooperación universidad-empresa persigue una visión de mediano plazo en la que la universidad comanda el desarrollo de un cluster tecnológico en la región.

En los últimos años diferentes grupos de investigación de la universidad participaron en redes de cooperación (con empresas, centros tecnológicos públicos y potenciales usuarios de las tecnologías) para la innovación en el marco de proyectos de investigación con aplicaciones tecnológicas concretas. Estas redes, enfrentaron por un lado, tensiones propias de las formas de articulación y coordinación con actores heterogéneos y por el otro, contextos de políticas nacionales de Ciencia y Tecnología diferentes. Es así que la interacción entre distintos agentes y organizaciones en los procesos de aprendizaje plantea tensiones entre el oportunismo derivado de la asimetría de intereses y el riesgo de encerramiento tecnológico. La trayectoria descripta a lo largo de la evolución de las redes muestra respuestas diversas según la forma en la que resuelven estas tensiones. La forma de resolver estas tensiones va a estar condicionada sin duda por el contexto de políticas de ciencia y tecnología, que será diferente según se trate de políticas bottom-up (desde abajo) en el que se apuntala el carácter espontáneo de los procesos de innovación, o por abordajes top-down (desde arriba) en el que los instrumentos de apoyo promueven la innovación en direcciones específicas a partir de criterios definidos a escala nacional.

En este contexto, en este artículo nos proponemos describir cómo la red enfrenta y resuelve las tensiones propias de los procesos de cooperación bajo dos esquemas diferentes de políticas bottom-up y top-down. Para ello se seleccionaron dos redes de cooperación para la innovación derivadas de proyectos científico-tecnológicos de grupos de investigación de UNSAM. La primera está dedicada al desarrollo de kits diagnósticos para salud humana y animal. Este grupo consolidó a lo largo de su trayectoria una plataforma tecnológica que combina bio y nanotecnología. La red se expandió o consolidó de la mano de las políticas del ministerio de ciencia y tecnología bajo un paradigma bottom-up. La segunda, dedicada al desarrollo y caracterización de microdispositivos electrónicos con aplicaciones aeroespaciales y seguridad, enfrentó un esquema de incentivos distinto, caracterizado por la demanda pública bajo un contexto de políticas del tipo top-down.

A fin de estudiar las formas en que coordinan los procesos de aprendizaje e innovación en estas redes de colaboración, se adopta el abordaje de las proximidades (BOSCHMA, 2005; BOSCHMA; LAMBOY, 1999; TORRE; RALLET, 1999, 2005). Este abordaje permite caracterizar, de forma estilizada, el conjunto 
de tensiones y las formas de coordinación que se deben considerar cuando una Universidad se propone impulsar las relaciones universidad - empresa en vistas a la constitución de un CCT. Desde esta perspectiva, todo proceso de aprendizaje e innovación involucra la combinación de distintos conocimientos distribuidos en distintas firmas y organizaciones que involucran problemas de incertidumbre y de coordinación. La proximidad entre los distintos actores resuelve estos problemas posibilitando la interacción, y luego la innovación y el aprendizaje.

En línea con esta literatura, nos preguntamos si la proximidad territorial es una o si es necesario aislar analíticamente otros tipos de proximidades. Como sostiene Boschma (2005) la proximidad geográfica no es condición necesaria ni suficiente para el desarrollo de un cluster de ciencia y tecnología (CCT). Desde esta perspectiva otras proximidades son aún más importantes con el fin de mejorar las comunicaciones y entre actores. Estas son la proximidad tecnológica o cognitiva, la proximidad organizacional, la proximidad social y la proximidad institucional.

En términos generales, Boschma (2005) propone que estas proximidades y la forma en que se eonjugan configuran pueden dar lugar a tensiones entre problemas de oportunismo y de encerramiento tecnológico. Bajos grados de proximidad plantean problemas de oportunismo e incertidumbre en las relaciones entre los agentes, limitando las posibilidades de interacción. Ciertos autores sostienen que, a mayor proximidad, mayor interacción, y por lo tanto mayor aprendizaje e innovación, un exceso de proximidad puede limitar o intensificar problemas de oportunismo y de asimetría de intereses entre los diferentes actores. La proximidad en sus diferentes dimensiones también puede dar lugar a problemas de encerramiento tecnológico o institucional (lock-in) como consecuencia de una alta especialización del cluster en una actividad o en una tecnología, se puede impedir la identificación de oportunidades y fuentes externas de conocimiento. Esta dinámica podría ser compensada a través de una menor proximidad en las otras dimensiones como es el caso de la proximidad social e institucional. Desde esta perspectiva estas tensiones entre oportunismo y encerramiento tecnológico pueden resolverse de dos maneras: por un lado, logrando niveles intermedios de proximidad, y por el otro, mediante configuraciones en las que altas proximidades en una(s) dimensión(es) puedan ser compensadas por bajas proximidades en otra(s) dimensiones.

Al igual que en gran parte de la literatura de clusters, uno de los principales problemas que enfrenta este tipo de análisis es la falta de consideración del rol que juega la articulación entre las dinámicas espontáneas que se dan en los clusters y los marcos generales de política industrial y tecnológica en los que los mismos se 
desarrollan. Esta problemática cobra relevancia en países de América Latina, en el que coexisten distintos abordajes de política industrial y tecnológica abriendo la posibilidad de distintas configuraciones de proximidades.

En línea con estas consideraciones generales, este trabajo se pregunta cómo las redes resuelven estas tensiones a lo largo de su trayectoria de manera diferente. Es posible establecer la hipótesis que aquellas iniciativas enmarcadas en políticas bottom-up, con una baja proximidad organizacional e institucional resuelven el riesgo de oportunismo a partir de una alta proximidad social. En contraste, casos caracterizados por estar guiados por políticas top-down presentan mayores riesgos de encerramiento tecnológico que se supera a partir de una disminución de la proximidad organizacional. Asimismo, desde una perspectiva dinámica, se plantea que es posible una convergencia entre las dos configuraciones polares descriptas aprovechando las ventajas respectivas de las mismas.

A fin de responder este interrogante este documento está organizado de la siguiente manera. En la primera sección, se presenta una breve discusión del marco conceptual. En la segunda sección, se presenta cómo la universidad se articula con las políticas nacionales de ciencia y tecnología. En la sección tres realizamos un estudio comparativo de dos redes de cooperación para la innovación en grupos de investigación que están en la base del cluster científico y tecnológico emergente. Para ello, luego de presentar la metodología empleada, analizamos dos proyectos de investigación y transferencia llevados a cabo por consorcios público-privados. Por último, se discuten los resultados obtenidos.

\section{Clusters de ciencia y tecnología: las proximidades como marco conceptual}

El enfoque francés de las proximidades desarrollado durante los años '90 (TORRE; RALLET, 1999) fue retomado por la literatura de la geografía económica evolucionista (BOSCHMA, 2005; BOSCHMA; LAMBOY, 1999; MARTIN; SUNLEY, 2010; KNOBEN, 2008; LAGENDIJK; OINAS, 2005) para identificar las dimensiones cognitivas o tecnológicas, organizacionales, sociales e institucionales que explican la coordinación en clusters y en especial en CCT, además de la proximidad geográfica. Boschma (2005) sugiere que dado que la innovación y el aprendizaje son actividades sujetas a problemas de incertidumbre y coordinación, la co-localización geográfica no es condición necesaria ni suficiente para el desarrollo de un CCT. No es necesario, ya que puede ser reemplazado por otros tipos de proximidad y no es 
suficiente debido a que la co-localización no asegura interacciones requeridas para la innovación. Es necesario considerar además la proximidad tecnológica, organizacional, social e institucional, que en distintas configuraciones facilitan y fomentan la búsqueda de soluciones a problemas comunes.

La proximidad tecnológica (o cognitiva) se refiere a la distancia entre bases de conocimiento complementarias entre distintas organizaciones. La geografía evolucionista, en confluencia con la literatura sobre innovación y aprendizaje tecnológico (NELSON; WINTER, 1982; COHEN; LEVINTHALL 1990; FREEMAN, 1991; LUNDVALL, 1992) coloca en un rol central el carácter localizado y heterogéneo de las bases de conocimiento como origen de los problemas de coordinación (ANTONELLI, 2000). Cuando la proximidad tecnológica es baja, los actores enfrentan problemas de capacidad de absorción, lo que dificulta la interacción, mientras que si la proximidad es demasiado elevada, se enfrenan riesgos de encerramiento tecnológico por carencia de nuevas fuentes de oportunidades y aprendizaje. En este sentido sostienen que la innovación se genera en presencia de un grado intermedio de proximidad entre las bases de conocimiento, con el fin de estimular el aprendizaje tecnológico (LIPPMAN; RUMELT, 1992; NOOTEBOOM, 2000) (ver Cuadro 1).

Por su parte, otros tipos de proximidades contribuyen a reducir la incertidumbre en el proceso de innovación y de aprendizaje tecnológico. En este sentido, es importante tener en cuenta la proximidad organizacional, que inspirada en la literatura neo-institucionalista se refiere al grado de control jerárquico sobre los diversos actores en oposición al mercado en el que el grado de autonomía de las organizaciones es elevado (WILLIANSON, 1975, 1979, 1985). Una proximidad elevada le resta flexibilidad a la red en el proceso de toma de decisiones y actúa contra los incentivos de los actores que la componen si sus objetivos no están alineados con aquellos que ejercen el control jerárquico. En el otro extremo, una baja proximidad da lugar a mayores conflictos y a conductas oportunistas. En línea con los aportes de autores japoneses que estudiaron la interpenetración entre el mercado y la jerarquía en las redes (BABA; IMAI, 1989), es posible identificar formas intermedias de proximidad organizacional que posibilitan asegurar el control por parte de las firmas de los flujos de conocimiento y aprovechar las ventajas dinámicas de la especialización en un CCT (ver Cuadro 1).

En tercer lugar, la proximidad social, entendida como el enraizamiento de las interacciones mercantiles y no mercantiles de un CCT en vínculos entre las personas más allá de la filiación institucional, puede actuar como un mecanismo de coordinación mediante la confianza (GRANOVETTER, 1985; UZZI, 1996). 
Para que la alta proximidad social no sea contraproducente generando efectos de encerramiento, las relaciones interpersonales de una comunidad deberían permanecer abiertas alcanzando un nivel intermedio de proximidad social (COWAN; JONARD, 2004; BRESCHI; LISSONI, 2001). En el otro extremo si la distancia social es baja, no pueden aprovecharse los aprendizajes basados en las interacciones debido a la baja confianza de los miembros entre ellos (ver Cuadro 1).

Por último, Boschma señala la proximidad institucional, basado en la relevancia de compartir un marco institucional común que restringen el comportamiento de los actores posibilitando la acción colectiva reduciendo los costos de deliberación y la incertidumbre de los procesos de innovación (EDQUIST; JOHNSON, 1997; HALL; SOSKICE, 2001; HOFSTEDE, 1991). La proximidad institucional refiere tanto a macro-instituciones que definen las reglas del juego a escala nacional como a hábitos y valores que pueden operar a escalas sub-nacionales. Al igual que en los demás casos, una institucionalidad muy fuerte o débil es contraproducente en la medida en que puede restringir el comportamiento de los agentes generando encerramiento institucional en un extremo o problemas de oportunismo en el otro (ver Cuadro 1).

CUADRO 1

Tensiones según el nivel de las proximidades

\begin{tabular}{|l|l|l|l|l|}
\hline \multirow{2}{*}{ Proximidad } & \multirow{2}{*}{ Dimensión } & \multicolumn{2}{|c|}{ Tensión } & \multicolumn{1}{c|}{$\begin{array}{l}\text { Solución a } \\
\text { la tensión }\end{array}$} \\
\cline { 3 - 5 } & & Alta proximidad & Baja proximidad & Proximidad Media \\
\hline $\begin{array}{l}\text { Tecnológica } \\
\text { o cognitiva }\end{array}$ & $\begin{array}{l}\text { Grado de similitud } \\
\text { de la base } \\
\text { tecnológica }\end{array}$ & $\begin{array}{l}\text { Encerramiento } \\
\text { tecnológico }\end{array}$ & $\begin{array}{l}\text { Baja capacidad de } \\
\text { absorción }\end{array}$ & $\begin{array}{l}\text { Convergencia } \\
\text { de bases de } \\
\text { conocimiento. }\end{array}$ \\
\hline Social & $\begin{array}{l}\text { Grado de } \\
\text { pertenencia a una } \\
\text { misma comunidad } \\
\text { o red social }\end{array}$ & $\begin{array}{l}\text { Redes cerradas } \\
\text { con riesgo de } \\
\text { baja racionalidad } \\
\text { económica }\end{array}$ & $\begin{array}{l}\text { Oportunismo, } \\
\text { falta de lazos de } \\
\text { confianza. Débiles } \\
\text { feedbacks de } \\
\text { conocimiento tácito }\end{array}$ & $\begin{array}{l}\text { Redes abiertas } \\
\text { con posibilidad de } \\
\text { entrada a nuevos } \\
\text { actores }\end{array}$ \\
\hline $\begin{array}{l}\text { Organiza- } \\
\text { cional }\end{array}$ & $\begin{array}{l}\text { Grado } \\
\text { centralización del } \\
\text { control y toma de } \\
\text { decisiones. }\end{array}$ & $\begin{array}{l}\text { Encerramiento e } \\
\text { inercia organiza- } \\
\text { cional Dificultad } \\
\text { frente a cambios. } \\
\text { Incentivos débiles. }\end{array}$ & $\begin{array}{l}\text { Oportunismo } \\
\text { y conflicto de } \\
\text { intereses. }\end{array}$ & $\begin{array}{l}\text { Organizaciones } \\
\text { intermedias con } \\
\text { formas hibridas de } \\
\text { control }\end{array}$ \\
\hline Institucional & $\begin{array}{l}\text { Grado de similitud } \\
\text { en las normas } \\
\text { reglas y hábitos }\end{array}$ & $\begin{array}{l}\text { Encerramiento } \\
\text { institucional/ } \\
\text { inercia institucional }\end{array}$ & $\begin{array}{l}\text { Oportunismo } \\
\text { y conflicto de } \\
\text { intereses. }\end{array}$ & $\begin{array}{l}\text { Reglas, y hábitos } \\
\text { comunes pero } \\
\text { abiertos a cambios } \\
\text { institucionales } \\
\text { graduales. }\end{array}$ \\
\hline
\end{tabular}

Fuente: Elaboración propia sobre base Boschma (2005). 
Esta perspectiva es útil para identificar las tensiones entre oportunismo y encerramiento tecnológico. Mayor proximidad en todas las dimensiones mejora las posibilidades de comunicación, circulación de la información y por lo tanto en la coordinación, pero al mismo tiempo aumenta el riego de encerramiento, ya sea tecnológico como institucional o social. Por otra parte, una menor proximidad es conducente a la introducción de novedad en el sistema, a riesgo también de generar mayor conflicto y facilitar comportamientos oportunistas.

La política pública debe cumplir el rol de amortiguar estos conflictos sin conducir a un encerramiento. Desde la perspectiva de la discusión teórica sobre políticas de promoción de clusters, las políticas bottom-up estarían orientadas a generar condiciones para la innovación a partir de bajas proximidades organizacionales e institucionales, manteniendo abierto el sistema a la introducción de novedad. Las políticas top-down, por su parte, favorecerían la comunicación y los procesos de coordinación a partir de altas proximidades, pero a riesgo de caer en encerramiento.

En las próximas secciones analizaremos si estos patrones diferentes de proximidades que surgen del marco conceptual se verifican en el caso de la UNSAM, teniendo en cuenta la forma en la cual cada caso analizado se articula con la política nacional de CyT y dentro de grandes proyectos estructurantes derivados de políticas verticales en sectores estratégicos.

\section{Potencial para la emergencia de un cluster de ciencia y tecnología en la UNSAM en el contexto de las políticas nacionales}

La UNSAM es una universidad pública que en un muy corto período de tiempo (fue fundada en 1992) ha logrado consolidar una base de conocimiento científico con fuerte potencial para el vínculo con el sector productivo a través de la transferencia tecnológica. Dicho proceso se enmarca en un importante impulso desde el Estado Nacional a la generación de oportunidades tecnológicas a partir del financiamiento de la infraestructura pública de investigación en las Universidades y en otros organismos nacionales de CyT.

El período en que se analiza la experiencia de la UNSAM se caracterizó por la maduración del diseño e implementación de tas políticas de apoyo a las capacidades tecnológicas en Argentina, con una ampliación en la gama de instrumentos, que buscaron pasar de un enfoque de "Fallas de Mercado" a otro basado en el apoyo a determinados aglomerados productivos, que generaron las condiciones para procesos de innovación y aprendizaje bottom-up (Lavarello y Sarabia, 2015). 
En forma paralela, Argentina puso en práctica "un conjunto de políticas con un abordaje top-down, en el que el Ministerio de Planificación (y en forma más incipiente el Ministerio de Defensa) impulsó las áreas satelital y nuclear sobre la base de las capacidades científicas y tecnológicas acumuladas por agencias estatales (Lavarello y Sarabia, 2015).

De esta manera, el período analizado se caracterizó por un regreso del apoyo estatal a la generación de capacidades tecnológicas desde dos perspectivas muy diferentes. Por un lado, un abordaje bottom-up en el que las políticas tecnológicas horizontales van asumiendo complejidad y selectividad, y por el otro, un abordaje top-down al final del período que marcan el regreso de los grandes proyectos nucleares y espaciales al centro de la política tecnológica.

La UNSAM es una de las pocas universidades que se insertará como integrante de ambas iniciativas. La UNSAM está rodeada de sedes de importantes instituciones de larga trayectoria en ciencia y tecnología del país. En primer lugar, está el Centro Atómico Constituyentes, de la Comisión Nacional de Energía Atómica (CONEA), con amplia trayectoria I\&D en tecnología nuclear y en actividades de formación (en vinculación con el Instituto Sábato de UNSAM). En segundo lugar, está la sede central del Instituto Nacional de Tecnología Industrial (INTI). Un instituto público dedicado a la I\&D, a la provisión de servicios, asesoramiento y calidad a empresas locales. En particular, en la sede central funciona el Centro de Micro y Nanoelectrónica, de importantes vínculos con la UNSAM. En tercer lugar, el Instituto de Investigaciones Científicas y Técnicas para la Defensa, dedicado a la I\&D en el área de defensa. En cuarto lugar, se localiza también Servicio Geológico Minero Argentino dedicado a generar información geológica-minera, territorial y ambiental. Estas instituciones junto a la UNSAM constituyen el Polo Tecnológico Constituyentes, que a través de un convenio formal se comprometen al trabajo conjunto.

\section{El caso de dos redes de colaboración universidad-empresas en UNSAM}

Como discutimos en la sección previa, la UNSAM está activamente inserta en la política de ciencia y tecnología nacional de Argentina. En particular a través de instrumentos de política nacionales como el FONARSEC - un fondo sectorial orientado a financiar proyectos de investigación principalmente en las áreas de Biotecnología, Nanotecnologías y Tecnologías de la información y comunicaciónque permitieron la creación de consorcios públicos-privados. Pero también a través 
de la colaboración con otras instituciones de ciencia y tecnología nacional, como la Comisión Nacional de Actividades Aeroespaciales (CONAE), en el contexto de políticas verticales en sectores estratégicos.

En línea con el interrogante planteado en este trabajo, las redes de colaboración universidad-empresa presentan distintas configuraciones de proximidades según el contexto de políticas en el que está inserta. Con el fin de identificar los elementos comunes y los propios de cada caso, en la presente sección describimos la evolución de dos redes de colaboración, cada una en un contexto de política distinto.

Se adoptó una estrategia de investigación basada en estudio de caso que busca indagar "cómo" se resuelven las tensiones en la emergencia de un CCT, donde no hay control por parte de los investigadores de los eventos analizados (YIN, 2003).

Las unidades de análisis son las redes de colaboración. La selección de las mismas estuvo basada en un criterio teórico, identificando dos casos polares de patrones de proximidades, uno correspondiente a una política bottom-up resultante de herramientas horizontales y otro asociado a políticas top-down. Esta selección nos permite lograr cierto grado de control sobre el efecto contextual de las dinámicas particulares. El análisis de los datos se basó en la técnica analítica de comparación de patrones (pattern matching) (YIN, 2003). Esta técnica permite comparar los patrones empíricos de las redes seleccionadas con el patrón teórico.

La información para la elaboración de los casos surge de distintas fuentes y su triangulación: (i) una serie de entrevistas semi-estructuradas que se realizaron a los directores de proyectos de investigación durante el segundo semestre del año 2014. Las mismas se basaron en cuestionarios no estructurados orientados a la identificación y caracterización de las interacciones en el marco de la constitución de redes de colaboración. Estas entrevistas se realizaron a diferentes actores de cada red, totalizando 20 entrevistas para ambas redes. (ii) La técnica de entrevistas fue complementada con observación en presentaciones de los grupos en los que los directores de proyecto detallaron el desarrollo y resultados de su área de investigación. Estas presentaciones se llevaron a cabo en el contexto de un programa del rectorado (Economía, Tecnología y Desarrollo) de la universidad de relevar capacidades en investigación y transferencia. (iii) Se recabaron fuentes documentales secundarias proveniente de los proyectos de investigación, memorias y documentos de auto-evaluación de la Universidad, noticias periodísticas, con el objetivo de complementar la información de las entrevistas e identificar puntos de conflicto. (iv) Por último, se volvieron a entrevistar durante el 2015 a los miembros de cada red para conocer la evolución de estos proyectos. 


\subsection{Convergencia entre nano y biotecnologías: plataforma para el diagnóstico de enfermedades infecciosas}

Esta red de cooperación surge en el marco de políticas horizontales (bottom-up) del MINCyT que subsidian el financiamiento de desarrollos conjuntos entre organismos públicos y pequeñas y medianas empresas. La misma tiene como objetivo valorizar las capacidades en biotecnología y nanotecnología. En una primera etapa, la red se orientó en el desarrollo de un dispositivo que integra un sensor nanoelectrónico con un reactivo biotecnológico y en una segunda etapa, se reformuló hacia kits de diagnósticos a partir de tiras reactivas.

\subsubsection{Nanobiosensores para el diagnóstico in situ}

Este proyecto, denominado NANOPOC, tuvo como et objetivo el desarrollo de un kit de diagnóstico in situ de enfermedades infecciosas (POC, del inglés Point Of Care), es decir sin que el diagnóstico se realice en un laboratorio clínico. El mismo permite detectar in situ una serie de enfermedades en animales y/o humanos, en un lapso de tiempo muy reducido y sin tener que transportar las muestras desde el lugar en el que las mismas fueron recogidas. Este desarrollo, como se discute en esta sección, constituirá el punto de partida para la creación de una nueva plataforma nano-biotecnológica.

El desarrollo involucra bases de conocimientos en biotecnología, nanotecnología y microelectrónica que si bien cuentan con una fuerte complementariedad, son muy disímiles evidenciando una baja proximidad tecnológica. Dada la dificultad de integrar ambas bases de conocimiento en una misma organización, el mismo involucró inicialmente tres centros investigación cada uno con capacidades en una de estas bases de conocimiento. Dos de estos centros son institutos tecnológicos que pertenecen al INTI (el Centro de Electrónica Microelectrónica y el Centro de procesos superficiales) y uno a la UNSAM con capacidades en biología molecular, el Instituto de Investigaciones Biológicas (IIB). El desarrollo de los antígenos estuvo a cargo del IIB mientras que el componente nanoelectrónico fue desarrollado principalmente por los equipos del INTI.

A pesar de la baja proximidad organizacional inicial, la interacción y la retroalimentación de conocimiento tácitos fue posible dado que los investigadores de los tres centros contaban con experiencia y relaciones de amistad previa. Muchos investigadores de ambos compartían tareas part-time de docencia e investigación sin 
dejar de pertenecer a sus instituciones de origen. Luego, la baja proximidad organizacional fue compensada inicialmente por una alta proximidad social que posibilitó ampliar el campo de conocimientos de cada organismo posibilitando importantes retroalimentaciones de conocimientos tácitos necesarias para combinar el antígeno con los dispositivos nano y micro electrónicos.

La alta proximidad social jugó un rol crucial en la primera etapa del proyecto a partir de la articulación informal con centros de investigación extranjeros. Una de las principales oportunidades que originaron el proyecto fue el acceso a un antígeno recombinante, una glicoproteína, a la que se accedió a partir de vinculaciones con investigadores radicados en la Universidad de Alberta (Canadá) con los que existía experiencia previa. El IIB había participado en las pruebas pre-clínicas para utilizar este antígeno como vacuna, que pese a no resultar exitosas abrieron la posibilidad de identificar el nuevo uso en diagnósticos.

Si bien la proximidad geográfica entre el campus de la UNSAM y el INTI constituye un factor que genera las condiciones para facilitar la interacción entre los científicos e ingenieros de las dos instituciones, es de particular importancia la pertenecía a una misma comunidad entre organismos, lo que permitió superar (o al menos postergar) los conflictos por la apropiación (oportunismo) o de encerramiento en un conjunto acotado de tecnologías.

Conforme fue avanzando el proyecto de la etapa de Investigación y Desarrollo (I\&D) al escalado se fueron incorporando nuevos actores y pasando de relaciones entre actores independientes basadas en la confianza y la experiencia previa hacia redes de cooperación formales (mayor proximidad organizacional). En una primera etapa, el proyecto involucra a la empresa ADEE - un desprendimiento del INTI especializado en el desarrollo de sistemas electrónicos aplicados a la salud - para fabricar los nano-sensores. En una segunda etapa del proyecto se incorporaron las empresas Biochemiq, PyME dedicada a la producción de proteínas recombinantes para aplicaciones veterinarias, y Agropharma, una empresa mediana instalada especializada en producción/formulación de productos farmacológicos para veterinaria.

Si bien la alta proximidad social logró resolver en una etapa inicial las tensiones asociadas a los conflictos de intereses, oportunismo y a la dificultad de complementar bases de conocimiento muy diferentes, con el avance del proyecto surgieron nuevas tensiones. El proyecto, que contaba con un plazo de cuatro años para llegar a la etapa pre-comercial, no llegó a comercializarse. Los principales obstáculos se evidenciaron en el pasaje a la etapa de producción en escala que estuvieron asociados por un lado, a las dificultades en el diseño de un componente microelectrónico lo 
suficientemente robusto para ser aplicado por personal no especializado y, por el otro, las dificultades propias del marco regulatorio que requería conocimientos clínicos.

Luego, la baja proximidad tecnológica entre campos de conocimiento muy disímiles y la distancia entre los criterios de eficacia técnicos con las regulaciones nacionales se agudizaron al avanzar "aguas abajo". La alta proximidad social lejos de resolver estas nuevas tensiones las profundizaba en la medida que las relaciones de amistad entre los investigadores dificultaban cambiar el diseño original y la desactivación del componente microelectrónico.

Frente a estas nuevas tensiones, los responsables del proyecto buscaron alternativas técnicas que evitaran el desarrollo de nanosensores, más robustos y fáciles de aplicar que derivó en el desarrollo de dos kits diagnósticos: uno basado en placas ELISA y otro en tiras reactivas, que actualmente produce una empresa spin-off universitario radicada en el campus (Chemtest). También han apostado a una mayor vinculación institucional con el sistema nacional de salud que en definitiva es el usuario de estos desarrollos.

\subsubsection{Sustitución de importaciones a partir de las necesidades del sector público en el sector salud: kit de diagnóstico temprano de E. Coli.}

La evolución de la red de colaboración previa se reconfigura dando lugar a esta nueva red. Esta red puede entenderse dentro de una mismała trayectoria, en el que sobre la base de los aprendizajes (sobre todo organizacionales e institucionales) realizados, es posible explotar la misma plataforma tecnológica para un nuevo uso. De hecho, una de las respuestas a los problemas técnicos y regulatorios fue el desarrollo de un nuevo producto a partir de parte de la tecnología original. El kit de diagnóstico temprano de Síndrome Urémico Hemolítico (SUH), causado por la Escherichia Coli, fue uno de los principales subproductos que permitieron valorizar la plataforma de nanobiosensores desarrollada para el diagnóstico in situ de enfermedades infecciosas (NANOPOC). Para ello se buscó articular el desarrollo original del cluster basado en una lógica institucional de mercado con las necesidades del sistema de salud nacional. Se apuntó a reducir la distancia entre las lógicas institucionales nacionales y los hábitos y valores que fundamentaron el consorcio original. El Síndrome Urémico Hemolítico (SUH) es endémico en la Argentina y constituye un problema de prioridad para el sistema de salud pública que será uno de los principales impulsores de este proyecto. La prioridad que este desarrollo tiene para el sistema de salud, interesado en un proceso de rápido diagnóstico, y 
la prevención que este mismo kit podría aportar en materia de prevención a través del diagnóstico en los alimentos, influye en que los plazos y los costos regulatorios sean mucho menores que en el caso de un (bio) fármaco.

Un aspecto relevante a señalar, es que frente a las dificultades que enfrentaba la configuración de proximidades previas, en este caso se va avanzar hacia una mayor proximidad tecnológica, organizacional e institucional disminuyendo la proximidad social. Este cambio busca la resolución de problemas técnicos y regulatorios identificados durante el proyecto previo. Esto involucra un cambio de unabordaje tedesde una política horizontal de tipo bottom-up a un mayor alineamiento con las prioridades de política de salud del Estado Nacional en un esquema orientad por "misiones". El Estado jugará desde el inicio un rol central no solo a partir del financiamiento sino como principal destinatario del kit diagnóstico. El proyecto surge de la iniciativa de un laboratorio público, el ANLIS-MALBRAN, motivado por las dificultades que enfrenta el sistema de salud para la importación de los insumos para los test y de las severas consecuencias en la salud de la población infantil de esta enfermedad. El principal motivo de la carencia de sistemas de diagnóstico eficientes en Argentina son los altos costos de los insumos importados en el marco de la restricción de divisas.

Desde la perspectiva de los problemas técnicos planteados en el proyecto previo, se buscó resolver el límite que planteó el diseño del componente de aplicación microelectrónico. La proximidad tecnológica aumentará limitándose a la base de conocimientos biotecnológica y nanotecnológica. En este nuevo proyecto el dispositivo en el que se brinda el diagnóstico es mucho más maduro que el nanosensor, basándose tanto en placas ELISA como en tiras reactivas (típicos en los tests de embarazo de uso doméstico) cuya tecnología no exigiría importantes inversiones en tetel desarrollo micro electrónico.

Desde el punto de vista de la proximidad organizacional, el proyecto asumió el arreglo institucional de una red semi-abierta que posibilitó modificar el concepto del proyecto de manera flexible. Además de la incorporación del laboratorio público ANLIS-MALBRAN como impulsor del proyecto desde la demanda, el reemplazo de la tecnología de nanosensores por las tiras y las placas ELISA requirió modificar la red de alianzas que incluyó la incorporación de la empresa especializada en biotecnología Inmunova, vinculada al Instituto Leloir (centro privado de I\&D), para el desarrollo de anticuerpos.

El proyecto avanzó hasta la etapa clínica y las pruebas han sido altamente satisfactorias. Dadas las características del producto (un kit de diagnóstico) - en el 
que se exigen solo buenas prácticas de manufactura, la validación del producto exigió menos pasos que en los medicamentos - dada la prioridad para el sector público en un esquema top-down, las etapas de aprobación no serían un obstáculo mayor para avanzar. La manufactura sería llevada adelante por el mismo ANLIS-MALBRAN en el marco de una estrategia pública de producción. El avance de dicho proyecto estuvo condicionado por la aún baja proximidad institucional, en el que el ritmo en la transferencia de tecnología de producción dados los tiempos administrativos en los mencionados organismos en el marco de reglas y hábitos diferentes. El riesgo de continuidad de este proyecto estuvo menos asociado a cuestiones de coordinación de las redes sino a una revisión profunda de la política sanitaria con la asunción de un nuevo gobierno en el que predomina una visión de mercado en las políticas públicas en el que los proveedores externos son priorizados vis á vis la sustitución de importaciones.

\subsection{Micro dispositivos electro-mecánicos (MEMs) en aplicaciones aeroespaciales}

Este caso involucra la conformación de una red - denominada nodo Nanotec - que se enmarca desde el principio en un abordaje de política top-down. Estos desarrollos tecnológicos dan respuesta a necesidades estratégicas nacionales con proyección a mercados internacionales. Los objetivos específicos responden a prioridades estratégicas, ya sea aquellas que surgen del Plan Espacial Nacional, sistemas para la seguridad interior, y a desarrollos innovadores para la Salud.

Esta experiencia se inserta dentro de una trayectoria mucho más amplia, instaurada desde la Comisión Nacional de Energía Atómica (CNEA), y luego la Comisión Nacional de Actividades Espaciales (CONAE), en el que el Estado Nacional desde el Ministerio de Planificación impulsó la emergencia de uno de los pocos espacios en los que el Sistema Nacional de Innovación de la Argentina ha logrado un desempeño competitivo a nivel internacional. Con el fin de avanzar en estos proyectos, buscó complementarse - con éxito parcial- el financiamiento de CONAE con la presentación a convocatorias de la Agencia Nacional de Políticas Científicas y Tecnológicas del MINCyT en el marco de instrumentos de apoyo de carácter horizontal.

La organización de la red se orienta a distintos proyectos partiendo de las aplicaciones en radares y buscando nuevos nichos. El primero de ellos, que dio origen a la red, es el desarrollo de una antena plana en banda X del tipo "phased 
array". A través de la reducción de tamaño - sus partes móviles miden fracciones de milímetro- masa y consumo de potencia, estas antenas posicionarían a la Argentina entre los primeros países en este campo. Tomando como punto de partida este desarrollo y sobre la base de las capacidades en nanotecnologías se constituye una plataforma tecnológica que posibilitó avanzar hacia otros desarrollos que van desde un olfateador (sniffer) que permite detectar y medir drogas y explosivos en aeropuertos y cárceles, hasta un nanobiosensor para el diagnóstico de enfermedades.

En línea con la trayectoria tecnológica en el sector aeroespacial el desarrollo antena plana en banda X del tipo "phased array", trata de un desarrollo incremental, que inspirándose en un dispositivo similar diseñado en los Estados Unidos, incorpora un nuevo microdispositivo electro mecánico MEM a ser utilizado en los satélites. En lugar de las antenas móviles, se diseña una antena experimental en "phased array" de lóbulos ordenados de cuatro por cuatro en una suerte de parrilla fija. Los MEMs actúan como switches que regulan la radiofrecuencia de distintos lóbulos. De esta manera, al ser fija, se logra el objetivo de bajo consumo energético y de potencia. Si bien se trata de una innovación incremental, la posibilidad de importar sustitutos es imposible por ser una tecnología de uso militar, siendo su potencial es muy elevado: desde el uso en satélites militares hasta usos civiles en salud.

En este proyecto se articulan fundamentalmente dos organismos, la Escuela de Ciencia y Técnica de la UNSAM y la CONEA, a la que se agregan distintos organismos y empresas vinculadas en forma directa a la CONAE. Su organización se basaba originalmente en una red jerárquica en la que la agencia estatal involucra$\mathrm{da}$ - la CONAE - centralizaba el control y seguimiento de los proyectos, dado el carácter sensible en términos espaciales y militares. No obstante, desde un comienzo los objetivos de autonomía económica y política se combinan con la búsqueda de éxito comercial de los proyectos.

La alta proximidad organizacional se combinaba con una alta proximidad institucional y social. La doble pertenencia de los investigadores a la Universidad y a la CNEA resultó en reglas y hábitos comunes desde el punto de vista del secreto y de los protocolos de investigación muy diferentes de las reglas de la academia y el sector privado. Adicionalmente, la pertenencia a una misma comunidad de físicos e ingenieros jugó un rol central desde su inicio. La idea del proyecto surge en el marco de relaciones de confianza entre los creadores de la Escuela de Ciencia y Técnica de la UNSAM y directivos de la CONAE. Las relaciones de confianza y la existencia de reglas y hábitos comunes resultaron cruciales para posibilitar las interacciones y las retroalimentaciones de conocimientos tácitos al mismo tiempo 
que evitaron los problemas de oportunismo en la apropiación de los resultados de la innovación.

A medida que se avanzó de la etapa de desarrollo a la de escalado, las relaciones jerárquicas se combinaron con un mayor grado de apertura mediante relaciones contractuales resultando en una proximidad organizacional intermedia. En este sentido la cadena de valor del proyecto involucra el desarrollo in house, incluyendo el diseño, la simulación y patentamiento, la externalización de la etapa de fabricación en una planta de fabricación de semiconductores dedicada (foundry) extranjera, y la caracterización a partir de capacidades nano y microelectrónicas realizada por la UNSAM y CONAE.

La externalización de la fabricación se basa en relaciones de confianza previa que permite compensar los riesgos de apropiación de tecnología en un campo tan sensible. Aspecto que justifica que uno de los objetivos del proyecto sea construir una planta piloto que permitiría fabricar en escalas chicas. Esta planta involucró la adquisición de equipamiento complementario al existente en los laboratorios de las instituciones integrantes de la asociación ad-hoc, para implementar en Argentina una unidad mínima de diseño, fabricación y caracterización de micro y nano dispositivos (MEMS y NEMS), que posibilitó generar localmente capacidad de manufactura de dispositivos.

Esta configuración de proximidades posibilitó el desarrollo de un nuevo proyecto que busca generar transistores de radiofrecuencia para usos militares con aviones sin señal. Se trata de un desarrollo que se articula en el Plan Espacial Argentino y que tiene como potenciales usuarios a la Comisión Nacional de Actividades Espaciales (CONAE), Invap y otras empresas que desarrollan equipos de radiofrecuencia en telecomunicaciones, radares y satélites.

Esto involucró la incorporación de investigadores repatriados de Alemania que tienen know how de fabricación. El diseño y los estudios teóricos de simulación de este primer transistor se hicieron en la Argentina y el primer prototipo se hizo en Alemania, que proveyó su la planta para fabricarlo. No obstante, la fabricación de los segundos prototipos busca realizarse en Argentina. Se encuentran entre los objetivos de los organismos participantes incubar una pequeña empresa de tecnología que produzca equipamiento para aplicaciones espaciales y de seguridad, y eventualmente otra empresa para aplicaciones en el área salud.

En resumen, este proyecto muestra una lógica muy diferente a la del resto de los proyectos porque desde su concepción se articuló con el Estado en el marco de objetivos nacionales estratégicos. Los riesgos de rigidez institucional son parcial- 
mente compensados por una fuerte flexibilidad basada en la organización en red y las relaciones de confianza entre los miembros de una comunidad de científicos con un objetivo de ciencia orientada a la solución de problemas. Es a partir de estas relaciones que se identifican nuevas oportunidades y que es posible establecer esquemas contractuales sin poner en riesgo el secreto de los proyectos.

\section{Discusión}

Ante la aparición de nuevos paradigmas tecnológicos que se caracterizan por ofrecer mayores oportunidades tecnológicas a partir de los descubrimientos científicos de la biología molecular y la nanociencia, el apoyo desde la política de Cy T y políticas verticales en sectores estratégicos del Estado Nacional a las capacidades científicas y tecnológicas de las universidades se convierte en una de las fuentes principales para la creación de agrupaciones de Ciencia y Tecnología. Este documento por un lado, permite validar la hipótesis del enfoque de proximidades. que frente a la baja proximidad tecnológica asociada a estas tecnologías no solo es importante la proximidad territorial, sino otras dimensiones como la proximidad tecnológica, organizativa, social e institucional. En particular, la red como forma de organización intermedia entre la integración vertical y las relaciones de mercado; la pertenencia simultánea a la comunidad científica y la participación en las comunidades de empresarios, la combinación de reglas basadas en la lógica privada, con el mantenimiento de otro tipo de normas para la toma de decisiones y que aseguren la reproducción de las capacidades básicas de investigación (publicaciones, conferencias, número de alumnos) y con mecanismos de evaluación que apoyen la transferencia de tecnología como criterio de éxito de los grupos. 
CUADRO 2

Comparación redes de CyT en el CCT de la UNSAM

\begin{tabular}{l|c|c|c|c}
\hline & $\begin{array}{l}\text { Kit de diagnóstico } \\
\text { 1: NANOPOC }\end{array}$ & $\begin{array}{c}\text { Kit de diagnóstico } \\
\text { 2: SUH }\end{array}$ & $\begin{array}{l}\text { Nanotec 1: MEMs } \\
\text { para aplicaciones } \\
\text { aeroespaciales }\end{array}$ & $\begin{array}{c}\text { Nanotec 2: } \\
\text { Radiofrec. } \\
\text { olfateadores, } \\
\text { nanocarriers }\end{array}$ \\
\hline $\begin{array}{l}\text { 1. Proximidad } \\
\text { tecnológica }\end{array}$ & \multicolumn{1}{c}{ Baja } & Intermedia & Intermedia & Baja \\
\hline $\begin{array}{l}\text { (Grado de similitud } \\
\text { de las bases de } \\
\text { conocimientos) }\end{array}$ & $\begin{array}{l}\text { Conocimientos } \\
\text { complementarios } \\
\text { pero disimiles: } \\
\text { nanotecnología, } \\
\text { biotecnología, } \\
\text { microelectrónica }\end{array}$ & $\begin{array}{l}\text { Conocimientos } \\
\text { complementarios } \\
\text { pero (relativamente) } \\
\text { disimiles: ADNr, } \\
\text { proteínas, reactivos }\end{array}$ & $\begin{array}{l}\text { Conocimientos } \\
\text { complementarios } \\
\text { yimilares: micro- } \\
\text { simatectrónica y }\end{array}$ & $\begin{array}{l}\text { Conocimientos } \\
\text { complementarios } \\
\text { pero disimiles: } \\
\text { nanotecnología, } \\
\text { biotecnología, } \\
\text { microelectrónica }\end{array}$ \\
\hline $\begin{array}{l}\text { 2. Proximidad } \\
\text { organizacional }\end{array}$ & \multicolumn{1}{c}{ Baja } & Intermedia & Alta & Intermedia \\
\hline $\begin{array}{l}\text { (Grado de Control } \\
\text { por la Jerarquía) }\end{array}$ & $\begin{array}{l}\text { Red semi-abierta } \\
\text { en consorcio univ.- } \\
\text { empresa, organismos } \\
\text { de CyT (INTI) }\end{array}$ & $\begin{array}{l}\text { Red semi-abierta } \\
\text { en consorcio } \\
\text { universidad-spin } \\
\text { offs- Organismos } \\
\text { salud pública }\end{array}$ & $\begin{array}{l}\text { Consorcio univ.- } \\
\text { organismos públicos }\end{array}$ & $\begin{array}{l}\text { Consorcio univ.- } \\
\text { organismos públicos } \\
\text { y acuerdos puntuales } \\
\text { con el extranjero }\end{array}$ \\
\hline
\end{tabular}

\begin{tabular}{|c|c|c|c|c|}
\hline $\begin{array}{l}\text { 3. Proximidad } \\
\text { social }\end{array}$ & Alta & Intermedia & Alta & Alta \\
\hline $\begin{array}{l}\text { (Confianza basada } \\
\text { en encastramiento } \\
\text { social) }\end{array}$ & $\begin{array}{l}\text { Conocimiento } \\
\text { previo y pertenencia } \\
\text { a misma comunidad }\end{array}$ & $\begin{array}{l}\text { Flexibilidad / } \\
\text { relaciones confianza } \\
\text { con actores sector } \\
\text { público de salud }\end{array}$ & $\begin{array}{l}\text { Conocimiento } \\
\text { previo y pertenencia } \\
\text { a misma comunidad }\end{array}$ & $\begin{array}{l}\text { Conocimiento } \\
\text { previo y pertenencia } \\
\text { a misma comunidad }\end{array}$ \\
\hline $\begin{array}{l}\text { 4. Proximidad } \\
\text { institucional }\end{array}$ & Baja & Intermedia & Alta & Alta \\
\hline $\begin{array}{l}\text { (Base común de } \\
\text { reglas, hábitos y } \\
\text { leyes que regulan } \\
\text { las relaciones entre } \\
\text { individuos y grupos) }\end{array}$ & $\begin{array}{l}\text { Reglas de } \\
\text { la academia } \\
\text { (publicación) y } \\
\text { mercado (DPI) }\end{array}$ & $\begin{array}{l}\text { Reglas de la } \\
\text { academia (publi- } \\
\text { cación) y ciencia } \\
\text { orientada (efecti- } \\
\text { vidad soluciones) }\end{array}$ & $\begin{array}{l}\text { Reglas de la ciencia } \\
\text { orientada hacia } \\
\text { transferencia } \\
\text { (efectividad de } \\
\text { soluciones) }\end{array}$ & $\begin{array}{l}\text { Reglas de la ciencia } \\
\text { orientada hacia } \\
\text { transferencia } \\
\text { (efectividad de } \\
\text { soluciones) }\end{array}$ \\
\hline $\begin{array}{l}\text { 5. Proximidad } \\
\text { geográfica }\end{array}$ & Alta & Alta & Intermedia & Baja \\
\hline (distancia) & $\begin{array}{l}\text { Empresas localizadas } \\
\text { en las cercanías } \\
\text { del campus y } \\
\text { Conurbano Norte }\end{array}$ & $\begin{array}{l}\text { Empresas localizadas } \\
\text { en las cercanías del } \\
\text { campus, Conurbano } \\
\text { Norte y CABA }\end{array}$ & $\begin{array}{l}\text { Empresas y organis- } \\
\text { mos localizados en } \\
\text { Conurbano Norte y } \\
\text { Patagonia }\end{array}$ & $\begin{array}{l}\text { Empresas y organis- } \\
\text { mos localizados en } \\
\text { Conurbano Norte y } \\
\text { Alemania }\end{array}$ \\
\hline $\begin{array}{l}\text { Orientación de } \\
\text { política }\end{array}$ & Bottom-up & $\begin{array}{c}\text { Bottom-up / top- } \\
\text { down }\end{array}$ & Top-down & $\begin{array}{l}\text { Top-down / } \\
\text { bottom-up }\end{array}$ \\
\hline Tensiones & $\begin{array}{l}\text { Baja racionalidad } \\
\text { económica del } \\
\text { proyecto por alta } \\
\text { proximidad social }\end{array}$ & $\begin{array}{l}\text { Riesgo de rigidez } \\
\text { institucional }\end{array}$ & $\begin{array}{l}\text { Riesgo de inercia } \\
\text { institucional y de } \\
\text { encerramiento (lock } \\
\text { in) tecnológico }\end{array}$ & $\begin{array}{l}\text { Riesgo de bajas } \\
\text { capacidades } \\
\text { de absorción } \\
\text { tecnológica }\end{array}$ \\
\hline $\begin{array}{l}\text { Resolución } \\
\text { de tensión } \\
\text { (encontradas en la } \\
\text { UNSAM) }\end{array}$ & $\begin{array}{l}\text { Mayor interacción } \\
\text { con el sector público } \\
\text { y mecanismos } \\
\text { de mercado para } \\
\text { regular las relaciones } \\
\text { internas }\end{array}$ & $\begin{array}{l}\text { Mecanismos } \\
\text { de incentivo de } \\
\text { mercado en la } \\
\text { comercialización }\end{array}$ & $\begin{array}{l}\text { Compensado } \\
\text { por relaciones de } \\
\text { confianza fuertes } \\
\text { y búsqueda de } \\
\text { creación de spin } \\
\text { off para diversificar } \\
\text { aplicaciones }\end{array}$ & $\begin{array}{l}\text { Compensado por } \\
\text { ampliación de la } \\
\text { red a socios de la } \\
\text { industria y otros } \\
\text { subsistemas de } \\
\text { política pública }\end{array}$ \\
\hline
\end{tabular}

Fuente: Elaboración propia sobre la base de entrevistas. 
El aporte del trabajo es mostrar que los distintos patrones de proximidad obedecen a distintos abordajes de política de CyT y políticas verticales. Esto es de particular importancia para países en desarrollo en los que existen capas geológicas de distintos abordajes de política coexistiendo enfoques bottom-up en el que predominan instrumentos horizontales que facilitan las interacciones sin mayor selectividad que la tecnología con abordajes de política top-down en el que los instrumentos son selectivos y asociados a desarrollos específicos enmarcados en misiones.

Como se puede apreciar en lo Cuadro 2, estos abordajes diferenciados dieron lugar a dos configuraciones polares de proximidades que replican el patrón que surge como proposición del marco conceptual de las proximidades.

En este sentido se validan este enfoque conceptual mostrando que frente a tecnologías genéricas como las biotecnologías y las nanotecnologías es posible identificar distintas formas de coordinación de los procesos de aprendizaje e innovación. La manera que resuelven las tensiones entre el oportunismo y el encerramiento tecnológico comprende por un lado el caso del NANOPOC en el que las proximidades sociales altas limitan el oportunismo propio de proximidades bajas en las dimensiones organizacionales e institucionales. Por otro lado, en el caso de los dispositivos MEMs, la alta proximidad institucional y social es compensada por una proximidad organizacional intermedia que permite abrir la red jerárquica evitando los problemas de encerramiento tecnológico.

Desde una perspectiva dinámica es posible establecer que la resolución de las tensiones en una etapa del desarrollo de ambas experiencias mediante una configuración dada de proximidades, puede dar lugar a nuevas tensiones que requieran nuevos cambios en las mismas. Este fenómeno se evidencia claramente en el caso de los kits de diagnóstico. Frente a la baja proximidad tecnológica entre tecnologías tan disímiles entre la microelectrónica y la biotecnología, la baja proximidad organizacional e institucional, la alta proximidad social logró resolver la tensión que se daba en términos de limitar los conflictos de intereses que podrían haber surgido si las relaciones hubieran sido estrictamente mercantiles. No obstante, la alta proximidad social resultó inconsistente con la identificación de nuevas formas de resolución de problemas técnicos y regulatorios. Una proximidad social intermedia posibilitó cambiar la tecnología y requirió también niveles intermedios de las otras proximidades.

Por su parte en el proyecto de MEMs, inicialmente las tecnologías involucradas eran complementarias y en cierto grado similares. En ese marco las proximidades organizacionales, institucionales y sociales eran altas asegurando el control interno de las interacciones y las retroalimentaciones. A medida que se avanzó en el de- 
sarrollo del proyecto original y surgieron nuevos proyectos con tecnologías cuyas oportunidades requirieron diversificar la base de conocimientos se disminuyó la proximidad tecnológica y se resolvieron problemas de encerramiento tecnológico. En ese marco, la red jerárquica cerrada avanzo hacia una red más abierta en la que participaron empresas extranjeras y de otros sectores en la etapa de fabricación. De esta manera se logró compensar la alta proximidad social e institucional propia de la industria aeroespacial y su diversificación hacia usos comerciales.

Como resultado de las diferencias existentes en los patrones de proximidades asociados a las diferencias en los abordajes de política, los grupos emergentes en UNSAM han logrado avanzar hacia una configuración similar en la que predominan proximidades intermedias a nivel organizacional y tecnológico. Cada experiencia demostró un proceso de aprendizaje institucional que permitió superar sus desventajas y sacar provecho de sus ventajas.

La perspectiva de una política bottom-up dio la posibilidad de explotar las altas oportunidades tecnológicas en un contexto de baja proximidad organizacional, pero enfrento desventajas en la dificultad de apropiar los resultados de la innovación, con riesgo de oportunismo. Estos inconvenientes fueron compensados por la proximidad social y la apertura de la red a los socios del sector público.

El enfoque de política top-down tuvo un alto riesgo de encerramiento presente en la incertidumbre de la demanda que implica bajos incentivos y problemas para la consolidación de la trayectoria tecnológica. Estos inconvenientes se han resuelto mediante la combinación de la proximidad social y una apertura de la red a otros tipos de empresas, en busca de una proximidad organizacional menos estricta.

Como resultado de estos procesos se avanzó en el desarrollo no solo de nuevos productos sino fundamentalmente de plataformas tecnológicas que muestran que la proximidad tecnológica es el resultado y no el punto de partida siendo la proximidad social la condición común que hizo posible la convergencia en un cluster de CyT como resultado de políticas bottom-up y top-down. 


\section{Referencias bibliográficas}

ANTONELLI, C. The economics of information networks. Amsterdam y Ney York: Elsevier Science, 1992.

. Collective knowledge communication and innovation: the evidence of technological districts. Regional Studies, v. 34, n. 6, p. 535-547, 2000. doi: 10.1080/00343400050085657 ARTHUR, W. B. Silicon Valley'locational clusters: when do increasing returns imply monopoly? Mathematical Social Sciences, v. 19, n. 3, p. 235-251, 1990.

ASHEIM, B. T.; COENEN, L. Knowledge bases and regional innovation systems: comparing Nordic clusters. Research Policy, v. 34, n. 8, p. 1173-1190, 2005.

BABA, Y.; IMAI, K. Systemic innovation and cross-border networks: the case of the evolution of the VCR systems. In: SCHERER, F.; PERLMAN, M. Entrepreneurship, technological innovation and economic growth. Ann Arbor: University of Michigan Press, 1992. p. 143-153.

BELL, M.; ALBU, M. Knowledge systems and technological dynamism in industrial clusters in developing countries. World Development, v. 27, n. 6, 1715-1734, 1999.

BIANCHI, C.; GRAS, N.; SUTZ, J. Make, buy and cooperate in innovation: evidence from Uruguayan manufacturing surveys and other innovation studies. National innovation surveys in Latin America: empirical evidence and policy implications. Santiago: ECLAC, 2011. p. 97-122.

BOSCHMA, R. Proximity and innovation: a critical assessment. Regional Studies, v. 39, n. 1, p. 61-74, 2005. doi: 10.1080/0034340052000320887

Boschma RA, Lambooy JG (1999) Evolutionary economics and economic geography. J Evol Econ 9:411-429. doi: 10.1007/s001910050089

BRESNAHAN, T.; GAMBARDELLA, A. Building high-tech clusters: Silicon Valley and Beyond. Cambridge: Cambridge University Press, 2004.

BRESNAHAN, T.; GAMBARDELLA, A.; SAXENIAN, A. Old economy inputs for new economy outcomes: cluster formation in the new Silicon Valleys. In: BRESCHI, S.; MALERBA, F. (Ed.). Clusters, networks and innovation. Oxforf: Oxford University Press, 2005. p. $113-134$.

CASSIOLATO J.; SHAPIRO, M.; LASTRES, H. Local system of innovation under strain: the impacts of structural change in the telecommunications cluster of Campinas, Brazil. International Journal of Technology Management, v. 24, n. 7, p. 680-704, 2002. 
COHEN, W. M.; LEVINTHAL, D. A. Absorptive capacity: a new perspective on learning and innovation. Administrative Science Quarterly, v. 35,n. 1, 128-152, 1990.

COLYVAS, J. A. From divergent meanings to common practices: the early institutionalization of technology transfer in the life sciences at Stanford University. Research Policy, v. 36, n. 4, p. 456-476, 2007. doi: 10.1016/j.respol.2007.02.019

COOKE, P. regional innovation systems, clusters, and the knowledge economy. ICC, v. 10, n. 4, p. 945-974, 2001. doi: 10.1093/icc/10.4.945

. Regional innovation systems: general findings and some new evidence from biotechnology clusters. The Journal of Technology Transfer, v. 27, n. 1, p. 133-145, 2002.

COOKE, P.; MORGAN, K. The creative milieu: a regional perspective on innovation. In: DODGSON, M.; ROTHWELL, R. The handbook of industrial innovation. Cheltenham: Edward Elgar, 1994. p. 25-32.

EDQUIST, C.; JOHNSON, B. System of innovation: overview and basic concepts. 1997.

FREEMAN, C. Networks of innovators: a synthesis of research issues. Research Policy, v. 20, n. 5, p. 499-514, 1991. doi: 10.1016/0048-7333(91)90072-X

GRANOVETTER, M. Economic action and social structure: the problem of embeddedness. American Journal of Sociology, v. 91, n. 3, p. 481-510, 1985.

HALL, P. A.; SOSKICE, D. Varieties of capitalism: the institutional foundations of comparative advantage. Oxford: Oxford University Press, 2001.

HOFSTEDE, G. Cultures and organizations: software of the mind. Intercultural cooperation and its importance for survival.. New York: McGraw-Hill, 1991.

KEEBLE, D.; WILKINSON, F. Collective learning and knowledge development in the evolution of regional clusters of high technology SMEs in Europe. Regional Studies, v. 33, n.4, p. 295-303, 1999.

KNOBEN, J. Firm mobility and organizational networks: innovation, embeddedness and economic geography. Cheltenham: Edward Elgar Publishing, 2008.

LAGENDIJK, A.; OINAS, P. Proximity, distance and diversity: issues on economic interaction and local development. Aldershot: Ashgate, 2005.

LAVARELLO, P.; SARABIA, M. La politica industrial en la Argentina durante la década de 2000. CEPAL, 2015 (Documento de Proyecto, n. 44).

LIPPMAN, S. A.; RUMELT, R. P. Demand uncertainty, capital specificity, and industry evolution. Ind Corp Change, v. 1, n. 1, p. 235-262, 1992. doi: 10.1093/icc/1.1.235 
LONGHI, C.; QUÉRÉ, M. Innovative networks and the technopolis phenomenon: the case of Sophia Antipolis. Environment and Planning C, v. 11, n. 3, p. 317-317, 1993.

LUNDVALL, B. National innovation systems - Analytical concept and development tool. Industry \& Innovation, v. 14, n. 1, p. 95-119, 2007. doi: 10.1080/13662710601130863 National systems of innovation. Anthem Press, 1992.

MASKELL, P. Towards a knowledge-based theory of the geographical cluster. Ind Corp Change, v. 10, n. 4, p. 921-943, 2001. doi: 10.1093/icc/10.4.921

MASKELL, P.; MALMBERG, A. Localised learning and industrial competitiveness. Cambridge Journal of Economics, v. 23, n. 2, p. 167-185, 1999. doi: 10.1093/cje/23.2.167

NELSON, R. R.; WINTER, S. G. An evolutionary theory of economic change. Harvard University Press, 1982.

NIOSI, J.; BANIK, M. The evolution and performance of biotechnology regional systems of innovation. Cambridge J Econ, v. 29, n. 3, p. 343-357, 2005. doi: 10.1093/cje/bei044

NOOTEBOOM, B.; VAN HAVERBEKE, W.; DUYSTERS, G. et al. Optimal cognitive distance and absorptive capacity. Research Policy, v. 36, n. 7, p. 1016-1034, 2007. doi: 10.1016/j.respol.2007.04.003

NOOTEBOOM, B. Learning and innovation in organizations and economies. Oxford: Oxford University Press, 2000.

RALLET, A.; TORRE, A. Is geographical proximity necessary in the innovation networks in the era of global economy? GeoJournal, n. 49, p. 373-380, 1999. doi: 10.1023/A:1007140329027 Saxenian A (1990). Regional networks and the resurgence of Silicon Valley. California Management Review 33, 89-112.

SAXENIAN, A. Regional advantage: culture and competition in Silicon Valley and Route 128. Harvard University Press, 1996.

SCHMITZ, H. On the clustering of small firms. IDS Bulletin, n. 23, p. 64-69, 1992. doi:10.1111/j.1759-5436.1992.mp23003012.x

STORPER, M.; VENABLES, A. J. Buzz: face-to-face contact and the urban economy. $J$ Econ Geogr, v. 4, n. 4, p. 351-370, 2004. doi: 10.1093/jnlecg/lbh027

TORRE, A.; RALLET, A. Proximity and localization. Regional Studies, v. 39, n. 1, p. 47-59, 2005. doi: 10.1080/0034340052000320842

TRIPPL, M.; TÖDTLING, F. Developing biotechnology clusters in non-high technology regions - The case of Austria. Industry and Innovation, v. 14, n. 1, p. 47-67, 2007. doi: $10.1080 / 13662710601130590$ 
UZZI, B. The sources and consequences of embeddedness for the economic performance of organizations: the network effect. American Sociological Review, v. 61, n. 4, p. 674-698, 1996. doi: $10.2307 / 2096399$

WILLIAMSON, O. E. Transaction-cost economics: the governance of contractual relations. The Journal of Law and Economics, v. 22, n. 2, p. 233-261, 1979. doi: 10.1086/466942 . The economic institutions of capitalism. Simon and Schuster, 1985. . Markets and hierarchies. New York, 1975.

YOGUEL, G.; BOCCHERINI, F. Hacia un modelo interpretativo de las actividades innovativas en las PyMEs: evidencias del caso argentino. In: KANTS, H. Desarrollo y gestión de PyMEs : aportes para un debate necesario. Buenos Aires: Universidad Nacional General Sarmiento, 1998.

Desarrollo del proceso de aprendizaje de las firmas: los espacios locales y las tramas productivas. Revista Desarrollo Económico, v. 41, abr.-jun. 2001. 